\title{
-to: Campbell-Stokes PL, Taylor BJ on behalf of the New Zealand Children's Diabetes Working Group (2005) Prospective incidence study of diabetes mellitus in New Zealand children aged 0 to 14 years. Diabetologia 48: 643-648
}

Received: 4 May 2005 / Accepted: 11 July 2005 / Published online: 30 September 2005

(C) Springer-Verlag 2005

\section{To the Editor:}

Campbell-Stokes et al. [1] recently described the incidence of type 1 and type 2 diabetes in New Zealand children aged 0-14 years for the years 1999 and 2000. The short time frame for this report is a major limitation, as has clearly been demonstrated by our longitudinal study over 30 years in Canterbury, New Zealand [2]. The aim of this note is to provide the balance of the incidence data that are available for New Zealand children and that were not acknowledged by Campbell-Stokes and colleagues [1].

Prospective ascertainment of all type 1 diabetes cases commenced in Canterbury in 1982. Incident cases presenting between 1970 and 1982 were ascertained retrospectively from hospital records. Primary ascertainment of incidence cases occurred through notification from the attending diabetes physician or paediatrician. All new cases developing diabetes in the $0-19$-year age group in the study area were either admitted to the regional hospital or attended acute intervention outpatient clinics at the same institution. The ascertainment of Canterbury cases is complete for the years 1970 to 2005 . At the last national census, the number of children under 15 years in Canterbury was 80,121 , with 108,384 children and adolescents under 20 years. The population of the Canterbury region was 398,157 , representing around $10 \%$ of the total New Zealand population. The total number of incident cases for the years 1970 to 2004 was 608 (315 males, 293 females). The lowest incidence $(95 \% \mathrm{CI})$ recorded was $2.40(2.72)$ cases per 100,000 person-years in 1970, with the highest incidence of

\footnotetext{
J. A. Willis $(\bowtie) \cdot R$. S. Scott

Lipid and Diabetes Research Group,

Christchurch Hospital,

Hagley Building,

Christchurch, New Zealand

e-mail: jinny.willis@cdhb.govt.nz

Tel.: +64-3-3640448

Fax: +64-3-3640457

B. A. Darlow

Department of Paediatrics,

Christchurch Hospital,

Christchurch, New Zealand
}

$28.60(10.48)$ cases per 100,000 person-years in 2004 . The incidence of type 1 diabetes in Canterbury between 1970 and 2005 is characterised by episodic peaks and troughs, giving a characteristic 'saw-tooth' graph (Fig. 1). These data suggest that comparisons of incidence between different regions should be integrated over relatively long time periods, to avoid misleading interpretations.

Since 1990, all incident cases aged 0 to 14 years have been submitted to the WHO DiaMond Study [3-5]. Canterbury is ranked internationally in the highest $10 \%$ of the incidence distribution for risk of childhood diabetes, and over the first decade of the study has moved from a medium-risk to a high-risk population with respect to type 1 diabetes [6].

The other longitudinal incidence register for childhood type 1 diabetes in New Zealand is held in Auckland. Incident cases in the Auckland region have been ascertained prospectively for longer than two decades and prospective data have also been submitted to the WHO DiaMond Study. The changes in the incidence of type 1 diabetes in children and adolescents diagnosed at younger than 15 years in Auckland and Canterbury were examined for the years 1977 to 1996 . The mean incidence rates for type 1 diabetes presenting prior to 15 years of age over the 20 -year period in Auckland and Canterbury were 11.61 and 16.44 cases per 100,000 persons per year respectively [7]. In Canterbury a very small number of cases were of non-European ethnicity. In Auckland, however, Maori and Pacific Island children represent a significant proportion of children newly presenting with type 1 diabetes. The mean incidence rates for type 1 diabetes in Auckland over the 20-year period in European, Maori and Pacific Island children were 15.41, 4.54 , and 5.75 cases per 100,000 persons per year, respectively [7]. Based on linear regression of the incidence data, significant increases in incidence of 0.65 cases per 100,000 persons per year were observed in Auckland children $(p<0.0001)$ and Canterbury children $(p<0.01)$ of European origin. In contrast, the incidence rates for Auckland children of Maori and Pacific Island ethnicity who presented with type 1 diabetes between 1977 and 1996 did not change significantly over time. The mean age at 


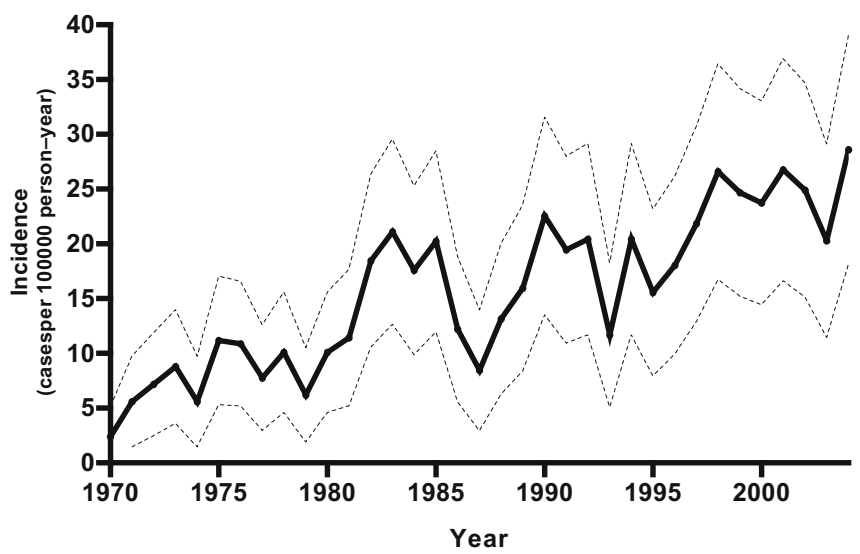

Fig. 1 The incidence of type 1 diabetes mellitus in children and adolescents aged less than 20 years in Canterbury, New Zealand, 1970-2004. Broken lines, 95\% CI

presentation with type 1 diabetes did not change significantly over time in either region, but the age distribution of cases varied significantly over time in Canterbury. While the overall incidence of type 1 diabetes is greater in Canterbury than Auckland, similar rates are observed in European children in both regions. In Auckland, children newly presenting with type 1 diabetes come from a greater diversity of ethnic groups, representing the diverse denominator population.

Seasonality in presentation with type 1 diabetes was observed in the Canterbury cohort and is most pronounced in male patients. A significant interaction was demonstrated between sex and season $(p<0.01)$ [2]. We have also demonstrated that the pattern of month of birth of Canterbury cases of type 1 diabetes shows a preponderance of births in spring and summer, whereas there is no significant rhythm in the pattern of live births in the general population [8]. A possible interpretation of these findings is that the autoimmune process is activated in utero or perinatally.

Increasing rates of presentation with type 1 diabetes in New Zealand children are not a regional phenomenon, and need to be addressed by appropriate forward planning of paediatric and adolescent health services in all regions of New Zealand. Campbell-Stokes and colleagues [1] provide a valuable snapshot of the national incidence of childhood diabetes in New Zealand for the years 1999 and 2000. A national prospective long-term register of diabetes presenting in New Zealand children and adolescents is required.

Acknowledgements This research was supported by the Health Research Council of New Zealand, the New Zealand Lottery Grants Board, and the R. G. Bell Charitable Trust.

\section{References}

1. Campbell-Stokes PL, Taylor BJ on behalf of the New Zealand Children's Diabetes Working Group (2005) Prospective incidence study of diabetes mellitus in New Zealand children aged 0 to 14 years. Diabetologia 48:643-648

2. Willis JA, Scott RS, Darlow BA et al (2002) The incidence of Type 1 diabetes diagnosed before age 20 in Canterbury, New Zealand over the last 30 years. J Pediatr Endocrinol Metab 15:637-643

3. WHO DiaMond Project Group (1990) WHO multinational project for childhood diabetes. Diabetes Care 13:1062-1068

4. WHO DiaMond Project Group (1991) Manual of methods of operation. Parts I-IV: incidence study. WHO, Geneva

5. WHO DiaMond Project Group (1992) Childhood diabetes, epidemics, and epidemiology: an approach for controlling diabetes. Am J Epidemiol 135:803-816

6. Karvonen M, Viik-Karjander M, Moltchanova E, Laporte R, Libman I, Tuomilehto J (2000) The incidence of type 1 diabetes worldwide. Diabetes Mondiale (DiaMond) Project Group. Diabetes Care 23:1516-1526

7. Willis JA, Scott RS, Darlow BA, Elliott RB, McGregor M (2004) Type 1 diabetes in children and adolescents: Auckland and Canterbury longitudinal cohorts. Proceedings of the New Zealand Society for the Study of Diabetes Annual Scientific Meeting, Auckland 2004

8. Willis JA, Scott RS, Darlow BA, Lewy H, Ashkenazi I, Laron Z (2002) Seasonality of birth and onset of clinical disease in children and adolescents $(0-19$ y) with type 1 diabetes mellitus in Canterbury, New Zealand. J Pediatr Endocrinol Metab $15: 645-647$ 